\title{
Comprehensive Evaluation and Analysis on Automobiles Performance Considering Objective Weights
}

\author{
Jian Zhang ${ }^{*}$, Xuexing Jiang, Liping Wang, Li Zou \\ School of Transportation and Automobile Engineering, Panzhihua University, Panzhihua 617000, China
}

\begin{abstract}
A fuzzy comprehensive evaluation method for vehicle performance based on variation coefficient method was proposed. The evaluation index system was constructed and the mathematical models of fuzzy comprehensive evaluation about vehicle performance were established. The method can cause evaluation bias because of the subjective weights of factors in traditional fuzzy comprehensive evaluation. In this paper, variation coefficient method which is objective weights was introduced to integrate with subjective weights from experts. In the end, the numerical examples were given, compared and evaluated the evaluation results with practical sales volume. The practical application showed that the evaluation method was effective and had good practicality.
\end{abstract}

\section{Introduction}

Automobiles gradually become daily-life necessities, and its vehicle performance has become key point for massive consumers, as a result, the scientific vehicle performance evaluation methods have become many scholars' goal [1]. Currently, there are many scholars have studied every major vehicle performance. It mainly concentrates on structure performance including handling stability, engine performance, automobile ride comfort, etc[2-3]. A variety of evaluation models are proposed and different evaluation methods are used to study vehicle each-part performance. However, fewer studies on vehicle performance. Due to multifarious and fuzzy vehicle performance indicators, it is an effective method to apply fuzzy comprehensive method to assess vehicle performance. When fuzzy mathematics theory is applied on comprehensive evaluation, the weights have a direct influence on the evaluation results [4-5].
Vehicle evaluation performance index parameters have been determined as soon as the vehicle get out of the car factory, which means that the effect of weights is objective, So objective weighting method should be applied. However, everyone's feeling vary from person preference to person on vehicle body aspect, it's appropriate to apply subjective weighting method. Integrating subjective and objective weights for evaluation index, finally more scientific vehicle comprehensive evaluation results were gained through fuzzy evaluation theory, which promoted manufacturers innovation of technology and provide reference for consumers.

\section{The determination of vehicle performance indicators systems and all indicators weights}

\footnotetext{
* Corresponding author: zhangjianpzh $@ 126 . c o m$
} 


\subsection{The vehicle-performance indicators systems}

In identifying evaluation index, choosing important, intuitive and specific indicators is convenient to compare differences among vehicles performance. The three indicators, space, configuration, process quality what consumers attach an importance to are chosen in the vehicle body. For the power assembly, what the

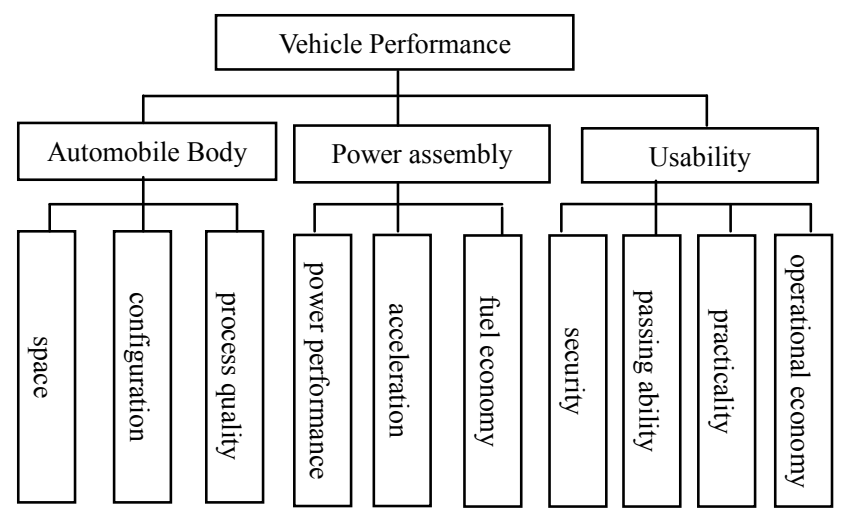

Fig.1. Automobile performance evaluation system structure

\subsection{Determine weights}

The determination of the weights can be divided into objective and subjective weighting method. Objective weighting method is a method which can directly obtain weights from each-index original information after a certain mathematics deal. Subjective weighting method refers to determining each-index importance weights after subjective analysis and study by professional knowledge and practical experience. Variation coefficient method is an objective weighting method which determine evaluation index weights according to variation degree of each evaluation index weights. Vehicle performance indicators are reflected by objective parameters. The index data that intuitive and variation degree is larger are suitable for Variation coefficient method. According to the characteristics of the performance evaluation index system and data statistics, power assembly and usability choose the variation coefficient method to determine weights. Vehicle body choose analytic hierarchy process(AHP) of subjective weighting method to determine weights.

The basic steps of variation coefficient method are: most direct way is to compare power performance, acceleration and fuel economy. The car is not simply walking implement. Therefore its security, passing ability, practicality and operational economy are an increasing importance when the usability index is determined. According to evaluation system principle, establish valuation index system as shown in Fig.1.

(1) Calculate the average of index $\overline{x_{f}}$ and variance $^{2}$

We suppose that there are q objects to evaluate, and among them have $\mathrm{p}$ indicators $x_{1}, x_{2}, \cdots, x_{p}$ to describe. Then, calculate the average of indicators $\overline{x_{f}}$ and variance $^{2} S_{f}$.

$$
\begin{aligned}
& \overline{x_{f}}=\frac{1}{q} \sum_{g=1}^{q} x_{g f} \\
& S_{f}^{2}=\frac{1}{q} \sum_{g=1}^{q}\left(x_{g f}-\overline{x_{f}}\right)^{2}
\end{aligned}
$$

(2) Calculate the each-index variation coefficient $v_{f}$.

$$
v_{f}=\frac{S_{f}}{\overline{x_{f}}}
$$

(3) Normalized processing for variation coefficient

$v_{f}$, get each-index weight.

$$
\omega_{f}=\frac{v_{f}}{\sum_{f=1}^{p} v_{f}}
$$

\section{The fuzzy evaluation method of vehicle performance}


Fuzzy evaluation is a method of using fuzzy mathematics theory to analyze and judge fuzzy phenomena. Fuzzy comprehensive evaluation judges fuzzy items in fuzzy environment for a particular purpose and takes into account multiple influence factors. Vehicle performance evaluation belongs to fuzzy problem for it involves multiple aspects, reflects more performance evaluation indicators, has complex influence factors. Thus fuzzy comprehensive evaluation method can be used to establish a multi-aspect ,multi-level and scientific system that can reflect the vehicle performance.

For vehicle performance fuzzy comprehensive evaluation problem, the mathematical model can be established as follows. Suppose the known factors affecting things set:

$$
X=\left\{x_{1}, x_{2}, \cdots, x_{n}\right\}
$$

$\mathrm{X}$ expressed as vehicle-performance various evaluation indicators set, is a single evaluation index. Evaluation set of thing is :

$$
U=\left\{u_{1}, u_{2}, \cdots, u_{m}\right\}
$$

$\mathrm{U}$ expressed as vehicle-performance various evaluation results set, is each-evaluation results. Statistics experiment or experts score are applied to establish distribution matrix of the weights between each factors.

\subsection{Establish the evaluation index weights set}

When we evaluate vehicle performance, each-evaluation index is given weight to fully reflect its important degree. The weights which consists of each weight coefficient are:

$$
W=\left\{W_{1}, W_{2}, \cdots, W_{n}\right\}
$$

In the formula (7), $\sum_{i=1}^{n} w_{i}=1$, Each weight should meet negative requirements.

\subsection{Construct comprehensive evaluation matrix of vehicle performance}

Determine the degree of membership of the evaluation object to each element in the evaluation set for the vehicles to be evaluated and fuzzy comprehensive evaluation matrix is constructed.

$$
R=\left[\begin{array}{cccc}
r_{11} & r_{12} & \cdots & r_{1 m} \\
r_{21} & r_{22} & \cdots & r_{2 m} \\
\vdots & \vdots & \cdots & \vdots \\
r_{n 1} & r_{n 2} & \cdots & r_{n m}
\end{array}\right]
$$

\subsection{Vehicle-performance fuzzy evaluation}

After $\mathrm{W}$ and $\mathrm{R}$ are determined, fuzzy decision integration $B$ is obtained by fuzzy synthesis operation $B$ $=\mathrm{W} \cdot \mathrm{R}=(\mathrm{b} 1, \mathrm{~b} 2, \ldots, \mathrm{bm})$, where symbol ". "is the operator notation. This is single factor evaluation result for the first level evaluation index. If those evaluation indicators are given weights $\mathrm{W}^{\prime}$ again, then secondary level comprehensive evaluation $\mathrm{C}=\mathrm{W}^{\prime} \cdot \mathrm{R}^{\prime}$ can be carried out. Similarly more hierarchy comprehensive evaluation can be continued. According to the specific results, the final evaluation conclusions are gained.

\section{The calculation and analysis of examples}

\subsection{Choose evaluation objects}

In establishing evaluation indicators, the seven secondary indicators including power performance, acceleration and fuel economy, as well as security, passing ability, practicality and operational economy can use corresponding data to reflect except the three secondary indicators of vehicle body. After dealing with and analyzing the statistical date of Autohome[6], finally engine horsepower values (ps), 0-100 km of per car acceleration time (s), various car models $100 \mathrm{~km}$ fuel consumption $(\mathrm{L})$, the minimum ground clearance $(\mathrm{cm})$, braking distance $(\mathrm{cm})$ of speed 100-0 km, trunk volume $(\mathrm{L})$, each routine maintenance costs are regarded respectively as judgment basis of seven indicators--power performance, acceleration, fuel economy, safety, passing ability, practicality, operational economy. Then get power assembly and usability, as shown in Table 1. 


\subsection{Calculate weights}

According to the formula (1) , (2) , (3) , (4), we gain corresponding secondary level indicators, as shown in Table $2 . \mathrm{W}_{21}=\left\{\begin{array}{lll}0.41 & 0.24 & 0.35\end{array}\right\}, \mathrm{W}_{22}=\left\{\begin{array}{l}0.34 \\ 0.0 .53\end{array}\right.$ $0.040 .090 .53\}$.

Three secondary indicators, space, configuration, process quality, corresponding to vehicle body are analyzed and calculated by AHP, and importance degree between them are compared, which can get weights $\mathrm{W}_{23}=\{0.15,0.56,0.29\}$. When consider three primary indicators, vehicle body, power assembly and usability performance, a plenty of investigations and researches are performed, and finished the results. The weights $\mathrm{W}^{\prime}=\{0.34,0.33,0.33\}$.

\subsection{The example of vehicle performance}

Now, take the joint venture vehicle models including Ashkenazi, U.S. and Japanese for example based on comprehensive evaluation of six different brand with same price, well-sold vehicle in the market. Six case on assessment are set up respectively expressed with $\mathrm{D}=\{\mathrm{D} 1, \mathrm{D} 2, \mathrm{D} 3, \mathrm{D} 4, \mathrm{D} 5, \mathrm{D} 6\}$. According to former evaluation index system and weights, experts are hired to score for each index. The score is shown as Table 3.

\subsection{The first level evaluation}

(1) Vehicle body

Through evaluating three indicators, space, configuration, process quality, vehicle body comprehensive evaluation are:

$$
\begin{aligned}
& \left.b_{11}=w_{21} \cdot\left[\begin{array}{l}
b_{21} \\
b_{22} \\
b_{23}
\end{array}\right]=(0.15,0.56,0.29) \cdot\left[\begin{array}{cccccc}
90 & 85 & 95 & 90 & 90 & 95 \\
85 & 95 & 95 & 90 & 80 & 85 \\
85 & 80 & 95 & 95 & 85 & 85
\end{array}\right] （ 9\right) \\
& =(85.75,89.15,95,91.45,82,95,86.5)
\end{aligned}
$$

(2) Power assembly

Through evaluating each-vehicle three indicators, power performance, acceleration and fuel economy, power assembly comprehensive evaluation is:

$$
\begin{aligned}
& b_{12}=w_{22} \cdot\left[\begin{array}{l}
b_{24} \\
b_{25} \\
b_{26}
\end{array}\right]=(0.41,0.24,0.35) \cdot\left[\begin{array}{cccccc}
85 & 95 & 85 & 90 & 80 & 90 \\
85 & 85 & 85 & 90 & 80 & 85 \\
85 & 80 & 90 & 100 & 90 & 85
\end{array}\right] \\
& =(85,87.35,86.75,93.5,83.5,87.05)
\end{aligned}
$$

(3) Usability Performance
Through evaluating each-vehicle four indicators, security, passing ability, practicality, operational economy, the comprehensive evaluation is:

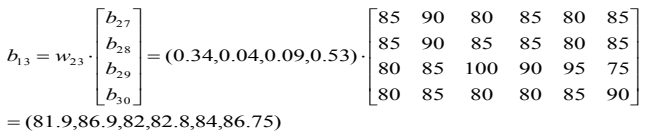

\subsection{The second level evaluation}

According to formula (9), (10), (11), the second level fuzzy comprehensive evaluation matrix of overall performance is:

$R^{\prime}=B=\left[\begin{array}{cccccc}85.75 & 89.15 & 95 & 91.45 & 82.95 & 86.5 \\ 85 & 87.35 & 86.75 & 93.5 & 83.5 & 87.05 \\ 81.9 & 86.9 & 82 & 82.8 & 84 & 86.75\end{array}\right]$

Combined with three primary indicators weights $\mathrm{W}$ $=\{0.34,0.33,0.33\}$, the secondary level evaluation result is:

$C=W^{\prime} \cdot R^{\prime}=(0.34,0.33,0.33) \cdot\left[\begin{array}{cccccc}85.75 & 89.15 & 95 & 91.45 & 82.95 & 86.5 \\ 85 & 87.35 & 86.75 & 93.5 & 83.5 & 87.05 \\ 81.9 & 86.9 & 82 & 82.8 & 84 & 86.75\end{array}\right]$

$=(84.232,87.8135,87.9875,89.272,83.478,86.764)$

\subsection{Discussion of results}

By analyzing fuzzy comprehensive evaluation results of vehicle performance we can get following conclusions:

From formula(13), we can conclude that the overall evaluation order of the six vehicle models is D4,D3,D2,D6,D1,D5. Thus comprehensive performance of D4 is the best, D3, D2 are basically equal, D6 is a little worse, and D1, D5 are the worst. D1, D5 should further improve in all aspects to achieve better quality.

At the same time, from the formula (9), (10), (11), the primary level evaluation results can be analyzed. It is easy to know which individual index is more outstanding or deficient among each-model body, power assembly and usability, which provide a reference for the modification of manufacturers.

\subsection{The analysis of comparing evaluation results with the actual sales}

Chinese domestic sales rank respectively was D1,D6,D3,D5,D2,D4 in 2015 by looking up the annual sales of six vehicle models in China. The sales of six vehicle models in North America were also looked up 
in 2015. Because data on D4,D5,D6 models were lacking in North America, for the another three models, the rank respectively was D2,D3,D1. To compare test performance results whether is positively related to actual sales, the Fig.2 was made.

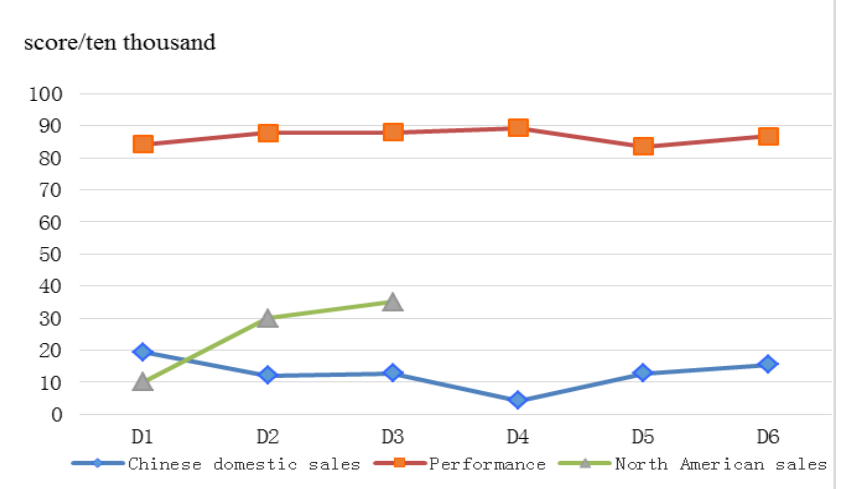

Fig.2.Comparison figure of evaluation and sales

As the Fig.2 shown, we can find out sales in North America is basically coherent with performance assessment rank, which proved that evaluation methods are effective and practical. But from the point of view of Chinese domestic sales, the pros and cons of the evaluation result is not positively related to actual sales except vehicle comprehensive performance factors. That is because of brands, products and personal preferences, etc. For example, the Ashkenazim joints venture vehicle models accumulated long-time public praise in Chinese automobile market, won high popularity. Its good image, fashion and generous appearance, accord with Chinese aesthetics, therefore, it is more praised by Chinese consumers. And partial Chinese consumers can not choose Japanese joints venture models because of personal preferences. Above factors affect all kinds of car sales in China, so the evaluation result is not positive correlation with the Chinese domestic actual sales. It's state that the domestic automobile consumption in China is not yet mature at present, and subjective factors of the car buyers have a greater impact.

\section{Conclusions}

A more scientific and rigorous vehicle performance evaluation of fuzzy comprehensive evaluation method based on variation coefficient was proposed in this paper. This method combined qualitative analysis with quantitative analysis, and considered comprehensively the influence of each evaluation index on vehicle performances. In order to solve subjectivity of determining weights in fuzzy comprehensive evaluation, we proposed the method that combined objective weight with the traditional subjective weight to determine weights, and testified its feasibility, effectiveness by practical examples. Its operation was simple, easy to achieve. Therefore, this method had good application value, not only provided a more actual new method for the automobile performances, also provided manufacturers with basis of improving and promoting automobiles performances.

\section{Acknowledgment}

In this paper, the research was sponsored by 2014 Research Program of Education Office of

Sichuan Province (Project No. 14ZB0409)

\section{References}

1. Daodong Liu, Tie Wang, Yi Gao, Hailin Li. Research on Evaluation Method of Vehicle Performance[J]. Mechanical Science and Technology for Aerospace Engineering, 2012 (3): 40-44.

2.Zhiqiang Zhang, Bin $\mathrm{Xu}$, Yongling He, ect. Engine Performance Evaluation Based on Analytic Hierarchy Process[J]. ACTA ARMAMENTARII, 2008(5):115-118.

3. Gang Chen, Weigong Zhang, Zongyang Gong, ect. Evaluation Method for Vehicle Shift feel Based on Evidence Theory and ANFIS[J]. Automotive Engineering, 2009(4): 19-23.

4.Jian Zhang, Xiaoying Liu, Jingdong Zhang, ect. Agriculture machinery safety and risk evaluation based on extension method[J]. Journal of Chinese Agriculture Mechanization, 2014(3):57-60, 66.

5. Caiyun Mao, Muchun Wu, Huazhong Lu. Fuzzy Synthesis Evaluation Method on Performance of Automobile Engine[J]. Journal of South China Agriculture University, 2010(3): 115-118.

6.Information on http://www. autohome. com. cn 
Table 1. parameters statistic of some meddle car table

\begin{tabular}{|c|c|c|c|c|c|c|c|c|}
\hline \multirow{2}{*}{ data } & \multicolumn{4}{|c|}{ Power assembly } & \multicolumn{4}{|c|}{ practicality } \\
\hline & Power & performance & acceleration & Fuel economy & Passing ability & security & practicality & Operational economy \\
\hline Passat & & 180 & 8.5 & 6.8 & 105 & 4133 & 490 & 798 \\
\hline Mondeo & & 203 & 8.1 & 7.9 & 124 & 3912 & 516 & 625 \\
\hline Accord & & 186 & 8.9 & 7.7 & 100 & 3887 & 512 & 307 \\
\hline Atenza & & 192 & 8.3 & 7.1 & 169 & 3896 & 483 & 543 \\
\hline Camry & & 184 & 9.1 & 7.8 & 159 & 3985 & 506 & 599 \\
\hline Magotan & & 160 & 8.9 & 7.8 & 114 & 4046 & 565 & 957 \\
\hline
\end{tabular}

Table 2. The calculation results of variation coefficient

\begin{tabular}{cccccccc}
\hline \multirow{2}{*}{ data } & \multicolumn{3}{c}{ Power assembly } & \multicolumn{5}{c}{ practicality } \\
\cline { 2 - 8 } & $\begin{array}{c}\text { Power } \\
\text { performance }\end{array}$ & Acceleration & Fuel & Passing & security & practicality & Operational \\
economy & ability & & & economy \\
\hline average & 184 & 8.6 & 7.5 & 129 & 3977 & 512 & 638 \\
standard deviation & 14.3 & 0.4 & 0.5 & 28.9 & 98 & 28.9 & 222.5 \\
variation coefficient & 0.0777 & 0.0465 & 0.0667 & 0.2240 & 0.0246 & 0.0564 & 0.3487 \\
weight & 0.41 & 0.24 & 035 & 0.34 & 0.04 & 0.09 & 0.53 \\
\hline
\end{tabular}

Table 3. The score and the weights of vehicle-performance evaluation indicators system

\begin{tabular}{ccccccccc}
\hline \multicolumn{2}{c}{ evaluation index system and weights } & weights & D1 score & D2 score & D3 score & D4 score & D5 score & D6 score \\
\hline Vehicle body & Space & 0.15 & 90 & 85 & 95 & 90 & 90 & 95 \\
0.34 & Configuration & 0.56 & 85 & 95 & 95 & 90 & 80 & 85 \\
& Process quality & 0.29 & 85 & 80 & 95 & 95 & 85 & 85 \\
Power assembly & Power performance & 0.41 & 85 & 95 & 85 & 90 & 80 & 90 \\
0.33 & Acceleration & 0.24 & 85 & 85 & 85 & 90 & 80 & 85 \\
& Fuel economy & 0.35 & 85 & 80 & 90 & 100 & 90 & 85 \\
Usability performance & Passing ability & 0.34 & 85 & 90 & 80 & 85 & 80 & 85 \\
0.33 & Security & 0.04 & 85 & 90 & 85 & 85 & 80 & 85 \\
& Practicality & 0.09 & 80 & 85 & 100 & 90 & 95 & 75 \\
\hline
\end{tabular}

